\title{
Measurement of differential cross sections in top pair production with the CMS detector
}

\author{
Ulrich Husemann* on behalf of the CMS Collaboration \\ Karlsruhe Institute of Technology \\ Institut für Experimentelle Kernphysik \\ 76131 Karlsruhe, Germany \\ E-mail: ulrich.husemann@kit.edu
}

\begin{abstract}
Measurements of differential cross sections for top quark-antiquark production are presented as a function of kinematic variables, jet multiplicities, and event-level observables. The measurements are based on proton-proton collision data recorded with the CMS detector during Run I of the CERN Large Hadron Collider at center-of-mass energies of $7 \mathrm{TeV}$ and $8 \mathrm{TeV}$.
\end{abstract}

The European Physical Society Conference on High Energy Physics

22-29 July 2015

Vienna, Austria

${ }^{*}$ Speaker. 


\section{Introduction}

Top quark-antiquark pairs $(t \bar{t})$ are produced in proton-proton collisions via the strong interaction. Due to the large $t \bar{t}$ production cross section at the Large Hadron Collider (LHC) at CERN, precision studies of differential cross sections have become feasible, allowing for detailed tests of quantum chromodynamics (QCD) at $\mathrm{TeV}$ energy scales. Detailed understanding of TeV-scale standard model processes such as $t \bar{t}$ production is also beneficial for the extraction of parton distribution functions, for Higgs boson physics, and for searches for physics beyond the standard model (BSM).

This article is organized as follows. In section 2 several $t \bar{t}$ differential cross section measurements are reviewed, followed by a discussion of jet multiplicity measurements in $t \bar{t}$ events in section 3. Measurements of event-level observables in $t \bar{t}$ events, such as transverse momentum sums, are presented in section 4. All of these measurements are based on proton-proton collision data taken with the CMS detector [1] during Run I of the LHC. The datasets correspond to integrated luminosities of approximately $5 \mathrm{fb}^{-1}$ at a center-of-mass energies of $\sqrt{s}=7 \mathrm{TeV}$ and $20 \mathrm{fb}^{-1}$ at $\sqrt{s}=8 \mathrm{TeV}$.

\section{Differential cross section}

The general strategy of the $t \bar{t}$ differential cross section measurements presented in this article is as follows. Events are selected in the $e / \mu+$ jets and in the dilepton $t \bar{t}$ decay channels, with criteria that ensure $t \bar{t}$ data samples with high purity. In particular, one or more of the jets have to be identified as coming from $b$ partons ( $b$-tagging). The $t$ and $\bar{t}$ kinematics are reconstructed from the decay products. Contributions of background processes such as single top production, $W / Z+j$ ets production, boson pair production, and QCD multijet production are estimated with data-driven techniques or taken from simulated data. After background subtraction, the data are corrected for detector acceptance, efficiency, and resolution effects using regularized unfolding techniques. These corrections are usually performed to the level of stable particles in the visible phase space, defined by requiring leptons and jets with minimum transverse momenta and within the angular acceptance of the CMS detector. If properties of the top quarks and antiquarks or the $t \bar{t}$ system are studied, all distributions are corrected to the level of top partons. In this case, the unfolding is performed both in the visible and in the full phase space of the event. The unfolded data is displayed as a normalized differential cross section to reduce systematic uncertainties. The data are compared with simulated data from various Monte-Carlo (MC) event generators and with theoretical calculations. The detailed procedures are described e.g. in $[2,3]$.

\subsection{Leptons and b-jets}

Differential cross sections as a function of the kinematic properties of leptons and $b$-jets are measured in the visible phase space of the CMS detector. Example distributions are displayed in figure 1, many more can be found in [3]. They are compared to the distributions generated with the MC event generators MADGRAPH [4] (interfaced to Pythia6 [5]), MC@ NLO [6] (interfaced to Herwig6 [7]) and PowHeg [8] (interfaced to Pythia6 and Herwig6). The lepton transverse momentum spectrum is slightly softer and the $b$-jet pseudorapidity distribution is slightly less central 
CMS, $19.7 \mathrm{fb}^{-1}$ at $\sqrt{\mathrm{s}}=8 \mathrm{TeV}$

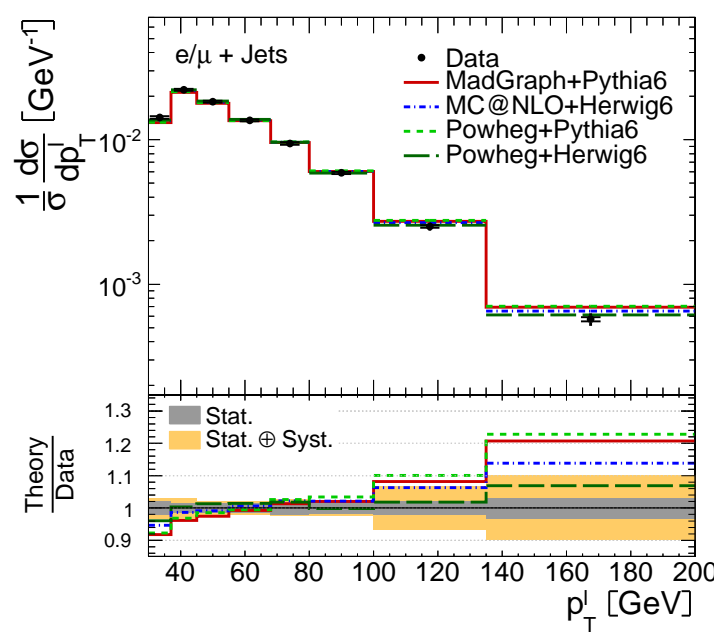

CMS, $19.7 \mathrm{fb}^{-1}$ at $\sqrt{\mathrm{s}}=8 \mathrm{TeV}$

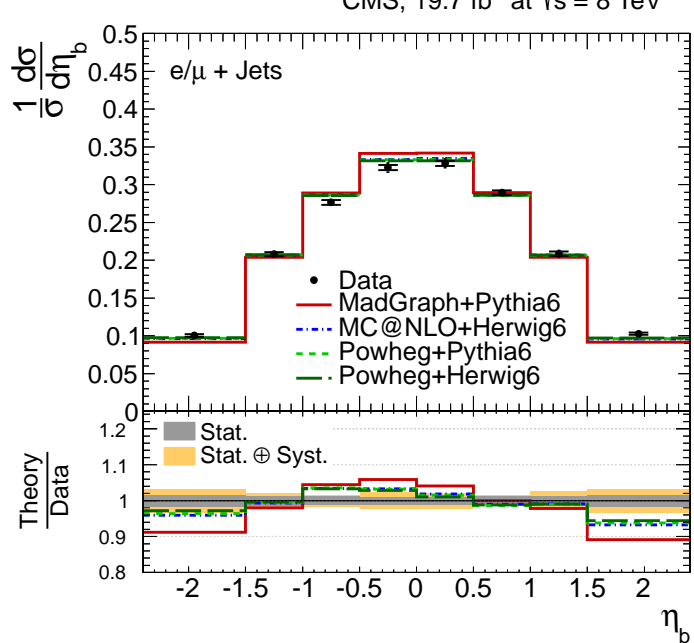

Figure 1: Examples of normalized differential cross sections as a function of lepton and b-jet kinematics at $\sqrt{s}=8 \mathrm{TeV}$. [3] . Left: lepton transverse momentum in $e / \mu+$ jets events. Right: b-jet pseudorapidity in dilepton events.

in data compared to simulations. Generally, the combination of POwHEG and Herwig6 describes all distributions well.

\subsection{Top quarks and $t \bar{t}$ system}

All differential distributions as a function of the top (anti)quark or $t \bar{t}$ system kinematics are corrected to the parton level, before the top decay, but after radiation. Example distributions are shown in figure 2. The distributions are compared to MC generators and also to calculations at nextto-leading order in QCD perturbation theory with resummation (NLO+NLL) [10] and approximate next-to-next-to-leading order (approx. NNLO) [9]. Generally good agreement is observed, however, the measured transverse momentum spectrum of top quarks is softer than predicted by most MC generators and the $t \bar{t}$ transverse momentum spectrum is not well described by the NLO+NLL calculation.

Various top differential cross section results obtained with the CMS detector at $\sqrt{s}=7 \mathrm{TeV}$ and $\sqrt{s}=8 \mathrm{TeV}$ as well as in the $e / \mu+$ jets and the dilepton decay channels have been compared and found to be consistent [3], as illustrated in figure 3.

\section{Jet multiplicity}

At the LHC additional QCD radiation is expected in more than half of the $t \bar{t}$ events. By measuring the jet multiplicity in $t \bar{t}$ events $[11,12]$, the validity and completeness of higher-order QCD calculations and MC event generators for multijet events can be studied. Important parts of the LHC physics program, such as associated $t \bar{t} H$ production, depend on the proper modeling of $t \bar{t}$ + jets production as an irreducible background. As for other differential cross section measurements, the jet multiplicity distribution is unfolded to particle level and compared to different MC event generators and to variations of the renormalization and factorization $\left(Q^{2}\right)$ scales, as well as 

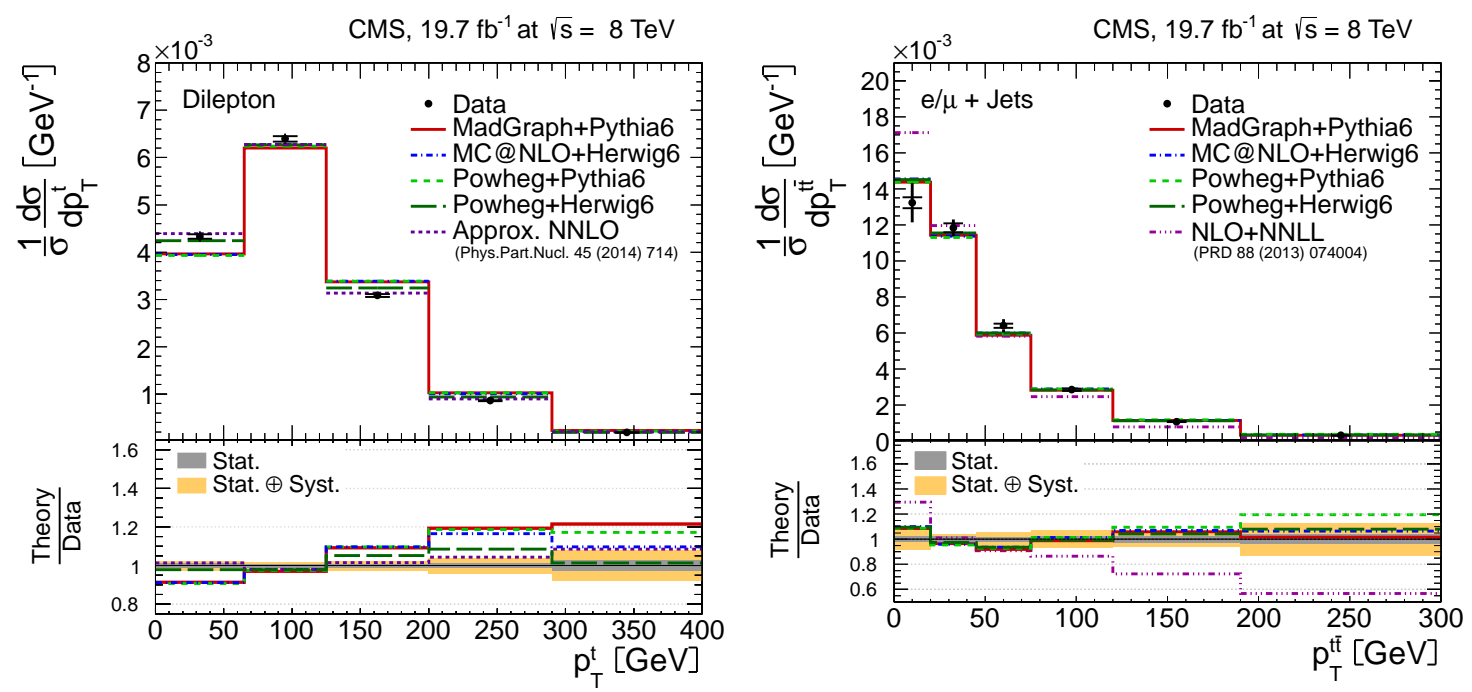

Figure 2: Examples of normalized differential cross sections as a function of top (anti)quark and $t \bar{t}$ system kinematics at $\sqrt{s}=8 \mathrm{TeV}$. [3]. Left: top quark transverse momentum in dilepton events. Right: $t \bar{t}$ transverse momentum in $e / \mu+$ jets events.

CMS, $19.7 \mathrm{fb}^{-1}$ at $\sqrt{\mathrm{s}}=8 \mathrm{TeV}$

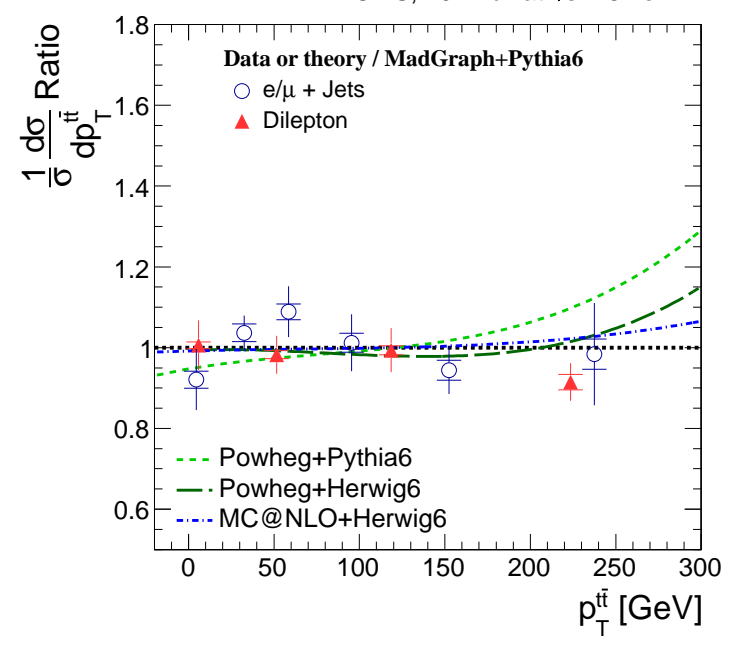

CMS, $5.0 / 19.7 \mathrm{fb}^{-1}$ at $\sqrt{\mathrm{s}}=7 / 8 \mathrm{TeV}$

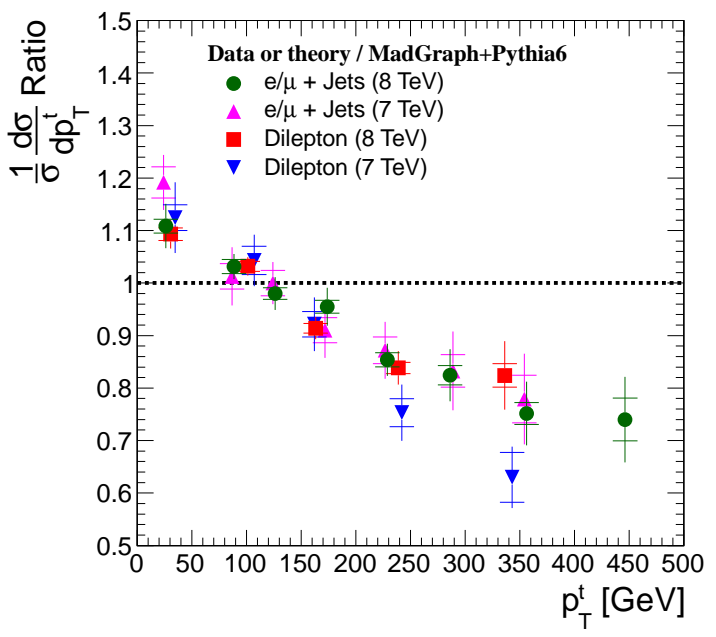

Figure 3: Comparison of differential cross section results [3]. Left: $t \bar{t}$ transverse momentum distribution compared between the $e / \mu+$ jets and the dilepton channels and MC generators. Right: top quark transverse momentum distribution compared between $\sqrt{s}=7 \mathrm{TeV}$ and $\sqrt{s}=8 \mathrm{TeV}$ and between the $e / \mu+$ jets and the dilepton channels. 

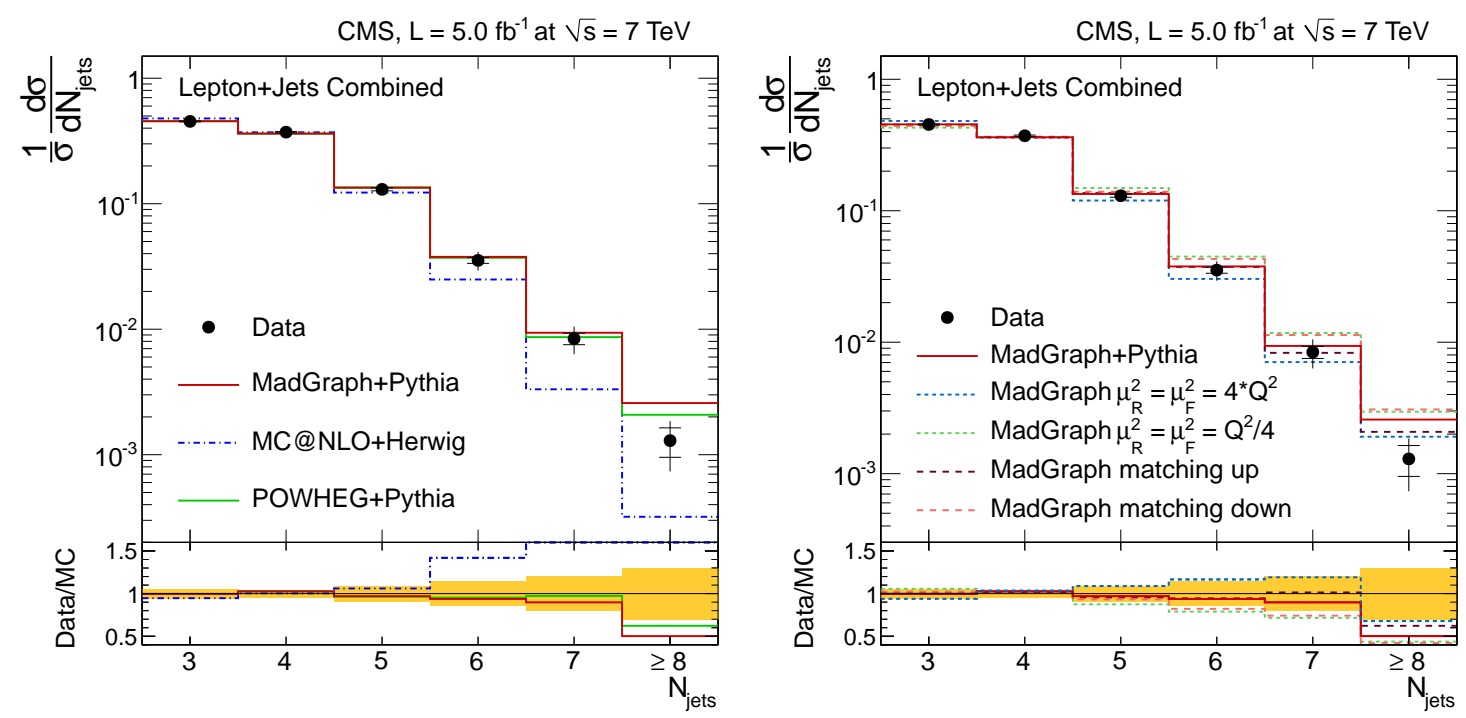

Figure 4: Normalized differential cross section as a function of jet multiplicity in the $e / \mu+j$ ets channel at $\sqrt{s}=7 \mathrm{TeV}$ [11]. Left: comparison with the MC event generators MADGRAPH, MC @ NLO, and POWHEG. Right: comparison with variations of the $Q^{2}$ scale and matching threshold in MADGRAPH.

variations of the matching threshold between radiation generated by the matrix element and the parton shower within MADGRAPH. As shown in figure 4, good agreement with MADGRAPH and PowHeG is observed, and the data precision is better than the scale variations by factors of four.

The CMS collaboration also explored alternative approaches to describe additional radiation in $t \bar{t}$ events. The multiplicity of additional jets, defined as jets not matched to partons from the top decay products, was determined by a maximum likelihood fit to data. The fraction of events in which observables related to additional radiation are below a certain threshold (e.g. transverse momenta of additional jets), called gap fraction, is also sensitive to the modeling of radiation in $t \bar{t}$ events. Selected results from both approaches are shown in figure 5. All distributions are well described within their uncertainties.

\section{Event-level observables}

Event level observables in $t \bar{t}$ events such as missing transverse energy and momentum sums were studied in $e / \mu+$ jets events at $\sqrt{s}=8 \mathrm{TeV}$ [13]. These observables are sensitive to rare processes, e.g. the associated production of $t \bar{t}$ pairs and $W, Z$, or Higgs bosons, as well as to BSM processes with lepton plus multijet signatures. In order to obtain a pure $t \bar{t}$ sample, $e / \mu+$ jets events are selected, requiring least two $b$-tagged jets. The sample is then split into subsets depending on the value of the event-level observable. In each subset the top content is extracted from a maximum likelihood fit to the pseudorapidity distribution of the $e$ or $\mu$, which is expected to be more central in $t \bar{t}$ events compared to background processes. The top content is then corrected for tauonic top decays and single top production and corrected for migration between subsets by regularized unfolding. As shown in figure 6, the event-level observables show good agreement with the MC event generators MADGRAPH, MC@NLO, and POWHEG within the uncertainties. 

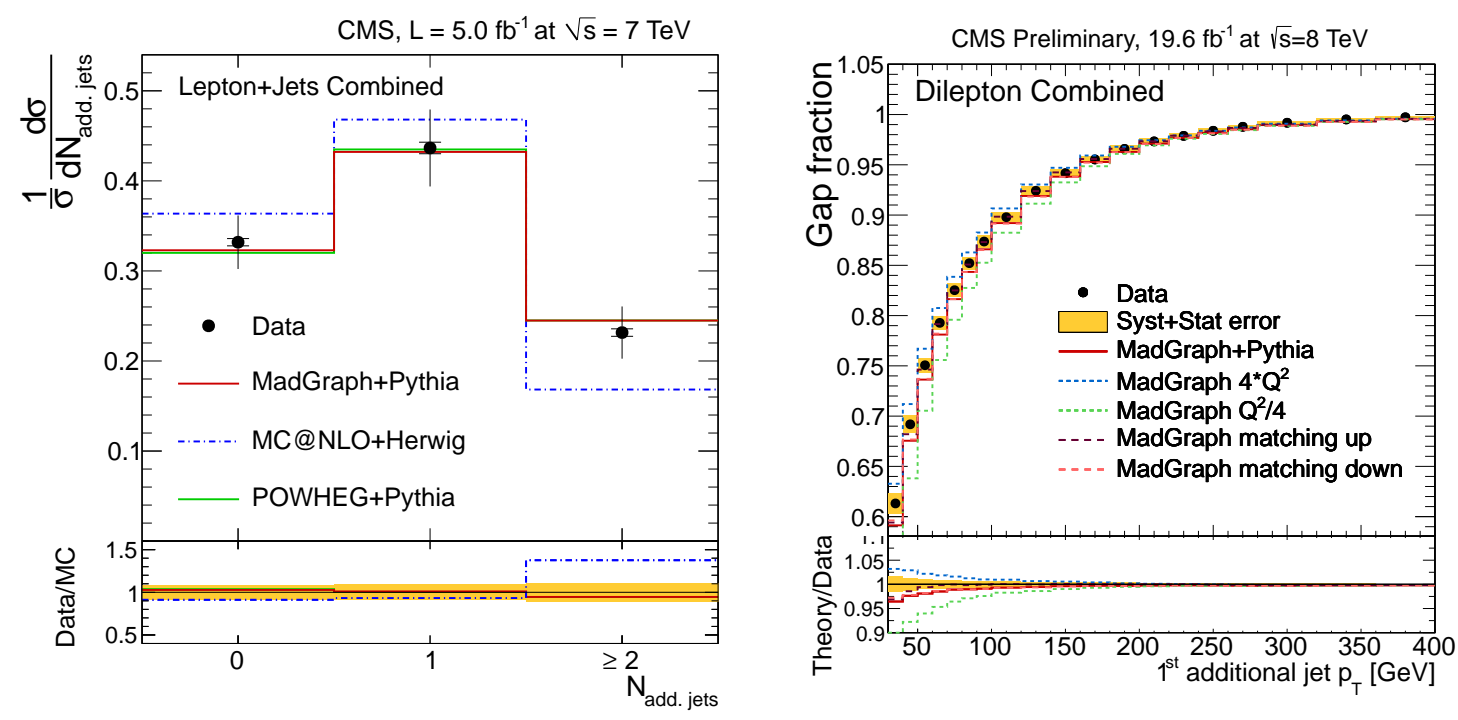

Figure 5: Alternative measures of additional radiation in $t \bar{t}$ events compared to the MC event generators MADGRAPH, MC@NLO, and POWHEG. Left: normalized differential cross section as a function of the number of additional jets in $t \bar{t}$ events in the $e / \mu+$ jets channel at $\sqrt{s}=7 \mathrm{TeV}$ [11]. Right: gap fraction as a function of the additional jet with the largest transverse momentum in the dilepton channel at $\sqrt{s}=$ $8 \mathrm{TeV}$ [12].
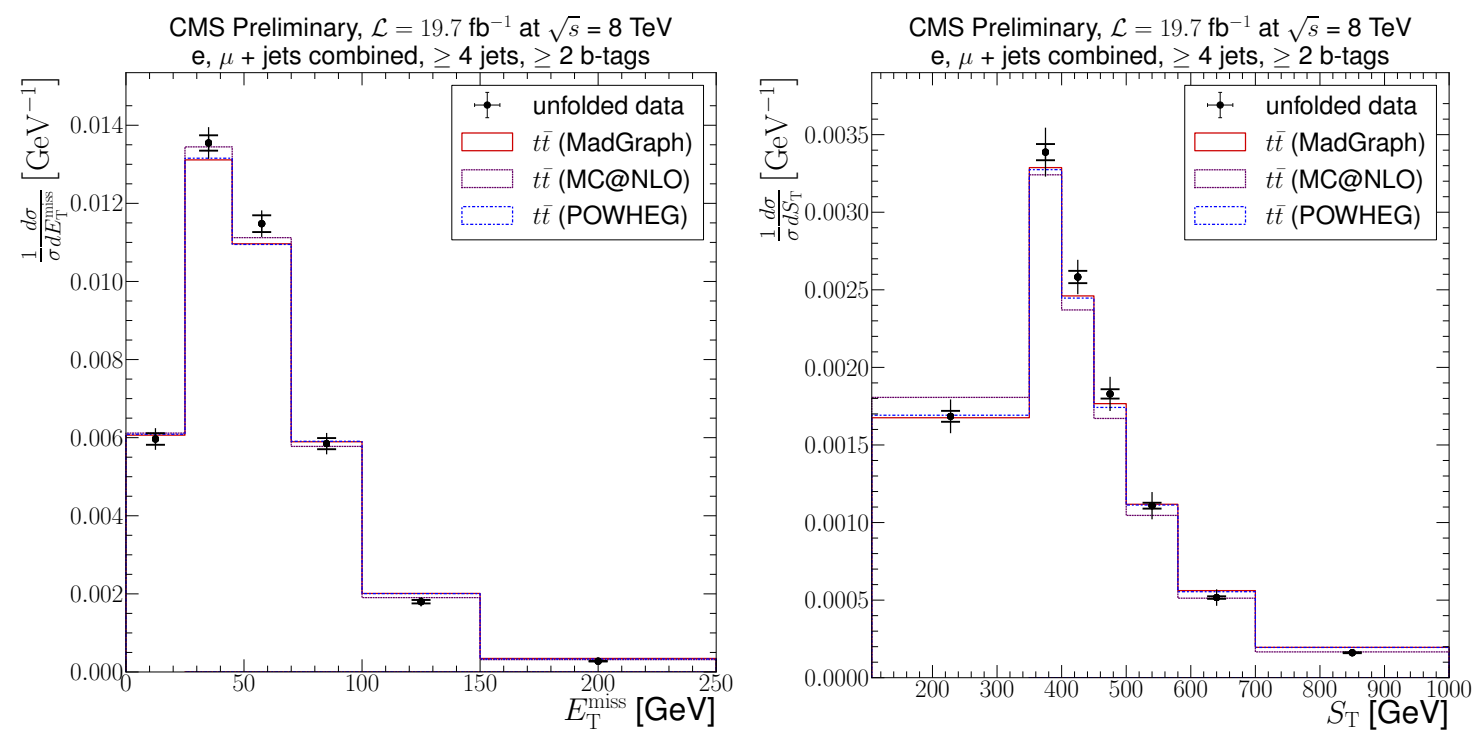

Figure 6: Examples of normalized differential cross sections as a function of event-level observables in $t \bar{t}$ events in the $e / \mu+$ jets channel at $\sqrt{s}=8 \mathrm{TeV}$ [13]. Left: missing transverse energy, $E_{\mathrm{T}}^{\text {miss }}$. Right: sum of the transverse momenta of all objects, $S_{\mathrm{T}}$. The data are compared to the MC event generators MADGRAPH, MC@NLO, and PowHEG. 


\section{Conclusions}

With Run I of the LHC, the physics of top quarks has made a big step towards precision measurements. An important cornerstone of this development is differential cross sections. At the CMS experiment, differential cross sections have been measured as a function of the $t \bar{t}$ system, the top (anti)quark and its decay products. To test the modeling of QCD radiation in $t \bar{t}$ events, jet multiplicities have been studied. Event-level observables sensitive to rare processes and BSM physics have also been extracted. Generally good agreement with standard MC event generators and higher-order calculations is found, the remaining discrepancies are being worked on together with the ATLAS experiment and the theory and MC event generator communities.

\section{Acknowledgments}

This work was supported by the German Bundesministerium für Bildung und Forschung (BMBF) under contract 05H15VKCCA.

\section{References}

[1] S. Chatrchyan et al. [CMS Collaboration], The CMS experiment at the CERN LHC, JINST 3 (2008) S08004.

[2] S. Chatrchyan et al. [CMS Collaboration], Measurement of differential top-quark pair production cross sections in pp colisions at $\sqrt{s}=7$ TeV, Eur. Phys. J. C 73 (2013) 3, 2339 [arXiv:1211.2220 [hep-ex]].

[3] V. Khachatryan et al. [CMS Collaboration], Measurement of the Differential Cross Section for Top Quark Pair Production in pp Collisions at $\sqrt{s}=8 \mathrm{TeV}$, arXiv:1505.04480 [hep-ex], submitted to Eur. Phys. J. C.

[4] J. Alwall, M. Herquet, F. Maltoni, O. Mattelaer and T. Stelzer, MadGraph 5 : Going Beyond, JHEP 1106 (2011) 128 [arXiv:1106.0522 [hep-ph]].

[5] T. Sjöstrand, S. Mrenna and P. Z. Skands, PYTHIA 6.4 Physics and Manual, JHEP 0605 (2006) 026 [hep-ph/0603175].

[6] S. Frixione and B. R. Webber, Matching NLO QCD computations and parton shower simulations, JHEP 0206 (2002) 029 [hep-ph/0204244].

[7] G. Corcella, I. G. Knowles, G. Marchesini, S. Moretti, K. Odagiri, P. Richardson, M. H. Seymour and B. R. Webber, HERWIG 6: An Event generator for hadron emission reactions with interfering gluons (including supersymmetric processes), JHEP 0101 (2001) 010 [hep-ph/0011363].

[8] S. Alioli, P. Nason, C. Oleari and E. Re, A general framework for implementing NLO calculations in shower Monte Carlo programs: the POWHEG BOX, JHEP 1006 (2010) 043 [arXiv:1002.2581 [hep-ph]].

[9] N. Kidonakis, NNLL threshold resummation for top-pair and single-top production, Phys. Part. Nucl. 45 (2014) 4, 714 [arXiv:1210.7813 [hep-ph]].

[10] H. T. Li, C. S. Li, D. Y. Shao, L. L. Yang and H. X. Zhu, Top quark pair production at small transverse momentum in hadronic collisions, Phys. Rev. D 88 (2013) 074004 [arXiv:1307.2464]. 
[11] S. Chatrchyan et al. [CMS Collaboration], Measurement of jet multiplicity distributions in $t \bar{t}$ production in pp collisions at $\sqrt{s}=7$ TeV, Eur. Phys. J. C 74 (2015) 3014, Erratum: Eur. Phys. J. C 75 [arXiv:1404.3171 [hep-ex]].

[12] CMS Collaboration, Measurement of the jet multiplicity in dileptonic top quark pair events at $8 \mathrm{TeV}$, CMS-PAS-TOP-12-041.

[13] CMS Collaboration, Measurement of MET and other global distributions in top pair events, CMS-PAS-TOP-12-042. 